\title{
Consumption of dairy foods in relation to impaired glucose metabolism and type 2 diabetes mellitus: the Maastricht Study
}

\author{
Simone J. P. M. Eussen ${ }^{1,2,3 *}$, Martien C. J. M. van Dongen ${ }^{1,3}$, Nicole Wijckmans ${ }^{1,3}$, Louise den Biggelaar ${ }^{1,2}$, \\ Stefanie J. W. H. Oude Elferink ${ }^{4}$, Cécile M. Singh-Povel ${ }^{4}$, Miranda T. Schram ${ }^{2,5}$, Simone J. S. Sep ${ }^{2,5}$, \\ Carla J. van der Kallen ${ }^{2,5}$, Annemarie Koster ${ }^{6}$, Nicolaas Schaper ${ }^{2,5}$, Ronald M. A. Henry ${ }^{2,5}$, \\ Coen D. A. Stehouwer ${ }^{2,5}$ and Pieter C. Dagnelie $e^{1,2,3}$ \\ ${ }^{1}$ Department of Epidemiology, Maastricht University, PO Box 616, 6200 MD, Maastricht, The Netherlands \\ ${ }^{2}$ School for Cardiovascular Diseases (CARIM), Maastricht University, PO Box 616, 6200 MD, Maastricht, The Netherlands \\ ${ }^{3}$ School for Public Health and Primary Care (CAPHRI), Maastricht University, PO Box 616, 6200 MD, Maastricht, The Netherlands \\ ${ }^{4}$ FrieslandCampina, Amersfoort, PO Box 238, 6700 AE, Wageningen, The Netherlands \\ ${ }^{5}$ Department of Internal Medicine, Maastricht University Medical Center +, PO Box 616, 6200 MD, Maastricht, The Netherlands \\ ${ }^{6}$ Department of Social Medicine, School for Public Health and Primary Care (CAPHRI), Maastricht University, PO Box 616, \\ 6200 MD, Maastricht, The Netherlands
}

(Submitted 22 June 2015 - Final revision received 25 November 2015 - Accepted 17 January 2016 - First published online 24 February 2016)

\section{Abstract}

Observational studies suggest an inverse association between total dairy product intake and diabetes risk. However, there is a lack of information on the relationship of specific dairy products with impaired glucose metabolism (IGM) and type 2 diabetes mellitus (T2DM). Individuals aged 40-75 years were recruited for the Maastricht Study. All the participants filled out a 253-food item FFQ, covering fifty specific dairy items that captured differences between full-fat, semi-skimmed and skimmed products, as well as fermented and non-fermented products. Glucose metabolism status was assessed by an oral glucose tolerance test, and participants were informed on their glucose metabolism status after returning the FFQ. Data of 2391 individuals were available to estimate OR (95\% CI) for IGM ( $n$ 470) and newly diagnosed (ND) T2DM ( $n$ 125), with adjustment for age, sex, BMI, physical activity, smoking status, education, energy intake and intakes of vegetables, fruits, meat and fish. For IGM, fully adjusted analyses revealed inverse associations, with OR comparing the highest with the lowest tertile of intake of $0.73(95 \%$ CI $0.55,0.96)$ for skimmed products and 0.74 (95\% CI $0.54,0.99)$ for fermented products. These dairy products were not associated with ND T2DM. In contrast, full-fat products were positively associated with ND T2DM (OR 2.01; 95\% CI 1.16, 3.47), whereas total dairy product intake was inversely associated with ND T2DM (OR 0.50; $95 \% \mathrm{CI} 0 \cdot 26,0 \cdot 93)$. In conclusion, individuals with a high consumption of skimmed and fermented products had lower odds of having IGM, and individuals with a high consumption of total dairy products had lower odds of having ND T2DM. High intake of full-fat products was not related to IGM but was positively related to ND T2DM.

Key words: Dairy foods: FFQ: Impaired glucose metabolism: Newly diagnosed type 2 diabetes mellitus: Observational studies

Type 2 diabetes mellitus (T2DM) is a metabolic disorder characterised by sustained hyperglycaemia. Hyperglycaemia in T2DM is due to insulin resistance, resulting from impaired sensitivity of tissues for insulin, and relative insulin deficiency. The prevalence worldwide of T2DM will increase from its present level of 225 million to as many as 552 million people by $2030^{(1)}$. Diabetes accelerates the development of other chronic diseases such as CVD, cognitive problems and decreased quality of life. Therefore, it is crucial to prevent diabetes and improve health status by identifying modifiable lifestyle factors including $\operatorname{diet}^{(2)}$.

Previous studies have demonstrated positive associations of circulating total free fatty acids with pancreatic $\beta$-cell dysfunction $^{(3)}$ and insulin resistance ${ }^{(4)}$. Other studies have suggested a negative association between dietary dairy-derived fatty acids and diabetes ${ }^{(5-7)}$. Up to now, five meta-analyses ${ }^{(8-12)}$ have summarised the results of prospective studies ${ }^{(12-15)}$ and randomised controlled trials ${ }^{(16,17)}$ on dairy product consumption and diabetes risk. Generally, these studies revealed an inverse relationship between consumption of total dairy products and T2DM.

Despite the presence of several meta-analyses on dairy consumption and $\mathrm{T} 2 \mathrm{DM}$, there is still a striking lack of knowledge regarding the role of specific dairy foods in the aetiology of T2DM, and studies have revealed mixed results on

Abbreviations: IGM, impaired glucose metabolism; ND, newly diagnosed; NGM, normal glucose metabolism; T2DM, type 2 diabetes mellitus.

* Corresponding author: S. J. P. M. Eussen, fax +31 43388 4128, email simone.eussen@maastrichtuniversity.nl 
the relationship between specific dairy products and diabetes. For example, most studies have observed inverse associations between yogurt consumption and T2DM risk ${ }^{(10-12,18)}$. In addition, cheese consumption was not associated with the incidence of the metabolic syndrome and/or $\mathrm{T}_{2} \mathrm{DM}^{(13)}$. However, cheese intake showed a, although not statistically significant, inverse association with diabetes incidence in the European Prospective Investigation into Cancer and Nutrition (EPIC) - InterAct Study ${ }^{(19)}$ and the Malmö Diet and Cancer cohort ${ }^{(20)}$. Furthermore, within Europe, substantial differences across countries exist with regard to consumption amounts of both total and specific dairy products ${ }^{(21)}$. For instance, total dairy product intake is relatively high in Spain and the Netherlands, whereas yogurt consumption is relatively high in Sweden and the Netherlands compared with other European countries $^{(21)}$. Moreover, none of the publications so far have included impaired glucose metabolism (IGM), which is associated with an increased T2DM risk as an outcome ${ }^{(22)}$. In line with the expected rise in incidence of $\mathrm{T} 2 \mathrm{DM}^{(23)}$, data from the 1999-2010 National Health and Nutrition Examination Survey revealed a $21 \%$ increase in IGM prevalence from 1999 through $2010^{(24)}$, whereas IGM prevalence will rise further from 344 million in 2010 to an estimated 472 million in $2030^{(25)}$. The aetiological hypothesis that dairy product intake is inversely associated with diabetes risk would be strengthened if associations between dairy product intake and IGM, a potential pre-stage of diabetes, have similar direction and magnitude compared with associations between dairy product intake and diabetes. The Maastricht Study is an extensive phenotyping study that focuses on the aetiology of T2DM, its classic complications and its emerging COmorbidities ${ }^{(26)}$. Within the framework of this cohort, we applied an extensive FFQ capturing fifty specific dairy products. The presence of data from an oral glucose tolerance test (OGTT), performed in all participants, also including individuals with normal glucose metabolism (NGM), allowed us to investigate associations of the consumption of specific dairy products with both IGM and newly diagnosed (ND) T2DM. In the present study, we included only individuals with IGM and ND T2DM because all individuals with pre-existing T2DM would have received advice on how to change their lifestyle, including their diet ${ }^{(27)}$.

\section{Methods}

\section{Study design, study area and population}

In this study, we used data from the Maastricht Study, an observational prospective population-based cohort study. The rationale and methodology have been described previously ${ }^{(26)}$. In brief, the study focuses on the aetiology, pathophysiology, complications and co-morbidities of T2DM and is characterised by an extensive phenotyping approach. Eligible for participation were all individuals aged between 40 and 75 years and living in the southern part of the Netherlands. Participants were recruited through mass media campaigns and from the municipal registries and the regional Diabetes Patient Registry via mailings. Recruitment was stratified according to known T2DM status for reasons of efficiency. The present report includes cross-sectional data from the first 3451 participants, who completed the baseline survey between November 2010 and September 2013. The examinations of each participant were performed within a time window of 3 months. All participants filled out a FFQ of the Maastricht Study after their first visit to the study centre and returned their completed FFQ by the third visit. Upon return of the FFQ, participants were informed about their glucose metabolisms status (e.g. normal glucose tolerance, IGM or diabetes). Participants who had previously been diagnosed with T2DM ( $n$ 883) were excluded from the present analyses as they already would have received dietary advice ${ }^{(27)}$, which likely would have changed their dietary habits. From the remaining 2568 participants, 117 individuals had not returned their FFQ, and sixty individuals had implausible energy intakes $(<3347$ or $>17573 \mathrm{~kJ} / \mathrm{d}(<800$ or $>4200 \mathrm{kcal} / \mathrm{d}$ ) for men and $<2092$ or $>14644 \mathrm{~kJ} / \mathrm{d}(<500$ or $>3500 \mathrm{kcal} / \mathrm{d}$ ) for women) ${ }^{(28)}$, and were therefore excluded as well. Finally, a total of 2391 participants (1796 with normal glucose tolerance, 470 with IGM and 125 with ND T2DM) were included in the present analyses.

The present study was approved by the institutional medical ethical committee (NL31329.068.10) and the Minister of Health, Welfare and Sports of the Netherlands, on the basis of the Health Council's opinion (Permit 131088-105234-PG). All the participants gave their written informed consent.

\section{Data collection}

Oral glucose tolerance test. To determine glucose metabolism status, all the participants (except those who use insulin) underwent a standardised seven-point OGTT after an overnight fast. Blood samples collected at baseline and 120 min after ingestion of a 75-g glucose drink were used to define diabetes status according to the World Health Organization 2006 criteria $^{(29)}$ into NGM, impaired fasting glucose (IFG), impaired glucose tolerance (IGT) and T2DM. In the present study, individuals with IFG or IGT were combined into one category - that is, IGM.

Dietary intake. Dietary intake was assessed by a tailor-made FFQ developed using the Dutch national FFQ tool ${ }^{(30)}$. The FFQ used in the Maastricht Study is one of the most extensive FFQ used so far in any study. It contains 101 questions on consumption over the last year, comprising twenty-three product groups and 253 items. The FFQ collected information on the intake of major food groups such as vegetables, fruits, meat, fish, dairy products and alcohol. Dairy product intake was appraised by fifty items covering milk (four items), chocolate milk (one item), coffee creamer (four items), ready-to-eat porridge (one item), cheese (nine items), yogurt (seven items), drink yogurt (three items), curd cheese (seven items), custard (six items), butter (two items), cream (four items) and ice cream (two items). Moreover, the FFQ captured differences between full cream, semi-skimmed and skimmed products, as well as fermented and non-fermented products (Table 1). Intake of energy and specific nutrients (SFA, Ca and lactose) was calculated using the Dutch NEVO food composition table, version $2011^{(31)}$.

Other relevant health parameters. All measurements were performed by trained research assistants during visits to the Maastricht Study research centre using standardised protocols. During study visits, office blood pressure $(\mathrm{mmHg})$ was measured, 
Table 1. Categorisation of fifty dairy food items into specific categories

\begin{tabular}{ll}
\hline Dairy products & Composition \\
\hline Total products & All dairy products mentioned below, plus coffee milk, chocolate milk and porridge \\
Full-fat products & Full-fat milk, Dutch cheese, foreign cheese, full-fat natural fruit yogurt and fruit curd cheese \\
Semi-skimmed products & Low-fat milk, Dutch cheese, foreign cheese, low-fat natural fruit yogurt and fruit curd cheese \\
Skimmed products & Skimmed milk, Dutch cheese, foreign cheese, skimmed natural fruit yogurt and fruit curd cheese \\
Fermented products & Whole-fat, low-fat and skimmed yogurts (both natural and with fruit) \\
& Whole-fat, low-fat and skimmed cheese (both Dutch and foreign, also including spreadable cheese) \\
Non-fermented, basic & Whole-fat, low-fat and skimmed curd cheese (both natural and with fruit), butter milk \\
Non-fermented, dessert & All types of milk and chocolate milk \\
& Whole-fat and low-fat cream, cooking cream, ice cream \\
Milk & Whole-fat, low-fat and semi-skimmed custard \\
Cheese, total & Whole-fat, low-fat and skimmed milk \\
& Dutch cheese: whole-fat, low-fat and skimmed cheese, also including spreadable cheese \\
Cheese, Dutch & Foreign cheese: whole-fat, skimmed and spreadable cheese \\
Cheese, foreign & Whole-fat, low-fat and skimmed cheese, also including spreadable cheese \\
Yogurt, total & Foreign cheese: whole-fat, skimmed and spreadable cheese \\
& Natural: whole-fat, low-fat and skimmed yogurt \\
Yogurt, natural & Fruit: whole-fat, low fat and skimmed yogurt, and skimmed yogurt with artificial sweeteners \\
Yogurt, fruits & Natural: whole-fat, low-fat and skimmed yogurt \\
Drink yogurt & Fruit: whole-fat, low-fat and skimmed yogurt, and skimmed yogurt with artificial sweeteners \\
Curd cheese, total & Natural, with sugar, with artificial sweeteners \\
& Natural: whole-fat, low-fat and skimmed curd cheese \\
Curd cheese, natural & Fruit: whole-fat, low-fat and skimmed curd cheese, and skimmed curd cheese with artificial sweeteners \\
Curd cheese, fruits & Natural: whole-fat, low-fat and skimmed curd cheese \\
\hline & Fruit: whole-fat, low-fat and skimmed curd cheese, and skimmed curd cheese with artificial sweeteners
\end{tabular}

and weight and height were measured to the nearest $0.1 \mathrm{~cm}$ and $0.5 \mathrm{~kg}^{(26)}$, respectively, to calculate BMI $\left(\mathrm{kg} / \mathrm{m}^{2}\right)$. Information on age and sex was extracted from study files, and information on education level (low, middle, high), physical activity, smoking habits (never, former, current) and self-reported medical history of CVD (yes, no) was derived from self-reported health questionnaires. Laboratory assessments included total cholesterol, LDL-cholesterol and HDL-cholesterol and TAG. Details about these measurements have been presented elsewhere ${ }^{(26)}$

\section{Statistical analysis}

Statistical analyses were performed using SAS version 9.3 (SAS Institute Inc.). Logistic regression models were fitted to estimate OR and their $95 \% \mathrm{CI}$ for the association between dairy product consumption and presence of IGM and ND T2DM. Crude models (model 1) were adjusted for age and sex. Fully adjusted multivariable models (model 2) were adjusted for sex, age, education (low, middle, high), BMI (continuous), physical activity (h/week), smoking (never, former, current) and intakes of energy, alcohol, vegetables, fruits, meat and fish. Associations were analysed with consumption of dairy product products expressed both as continuous measures (per serving for specific dairy products, and per $100 \mathrm{~g}$ increment for combined dairy categories) and as categorical variables, with tertile cut-off points based on intake of the NGM participants in our study population. All tests were two-tailed, and $P$ values $<0.05$ were considered statistically significant.

\section{Results \\ Characteristics of study population}

Of the 2391 participants included in this study, 1796 individuals had NGM, 470 had IGM and 125 were ND with T2DM.
The mean age was 59.9 (sD 8.2) years and 50.9\% were men. Altogether, $12.6 \%$ of the participants were current smokers and $17.0 \%$ reported a medical history of CVD. Compared with individuals with NGM, individuals with IGM or ND T2DM were more frequently male, were older and had higher BMI, lower physical activity, higher blood pressure and a higher prevalence of CVD. Moreover, individuals with IGM or ND T2DM tended to have lower intakes of fruits and vegetables and higher intakes of meat, whereas fish consumption was similar across subgroups of glucose metabolism status (Table 2).

\section{Intake $(\mathrm{g} / \mathrm{d})$ of dairy products}

The mean intake of total dairy products was 159 (SD 137) g/d in individuals with normal glucose tolerance compared with an average intake of 119 (sD 93) g/d in individuals with ND T2DM ( $P_{\text {for difference }}<0 \cdot 01$ ). Cheese was consumed by all participants and yogurt by approximately $80 \%$. Just over $50 \%$ of the total study population consumed milk and approximately $40 \%$ consumed butter. Butter milk, drink yogurt and curd cheese were consumed by a smaller proportion of the participants $(<35 \%)$. The consumption of milk was essentially the same for NGM, IGM and ND T2DM. The most pronounced differences across groups of glucose metabolism status were observed for the amount of consumption of yogurt, fermented, skimmed and semi-skimmed products and for total dairy product intake, with the highest intakes in individuals with NGM and the lowest intakes in individuals with ND T2DM (data not shown, online Supplementary Table S1).

Individuals in the highest tertile of yogurt intake $(\geq 63 \mathrm{~g} / \mathrm{d})$ had higher physical activity levels, were less often current smokers and consumed more fruits and vegetables and less meat compared with individuals in the lowest tertile of yogurt intake $(\leq 10.5 \mathrm{~g} / \mathrm{d})$. Similar patterns were observed for individuals 
Table 2. Population characteristics

(Mean values and standard deviations)

\begin{tabular}{|c|c|c|c|c|c|c|}
\hline & \multicolumn{2}{|c|}{ NGM $(n$ 1796) } & \multicolumn{2}{|c|}{ IGM ( $n$ 470) } & \multicolumn{2}{|c|}{ ND T2DM ( $n$ 125) } \\
\hline & Mean & SD & Mean & SD & Mean & SD \\
\hline \multicolumn{7}{|l|}{ Demography } \\
\hline Age (years) & $58 \cdot 1$ & 8.09 & 61.8 & 7.45 & $63 \cdot 3$ & 7.44 \\
\hline Sex (\% male) & \multicolumn{2}{|c|}{$45 \cdot 1$} & \multicolumn{2}{|c|}{54.9} & \multicolumn{2}{|c|}{$62 \cdot 8$} \\
\hline \multicolumn{7}{|l|}{ Lifestyle } \\
\hline \multicolumn{7}{|l|}{ Smoking (\%) } \\
\hline Never & \multirow{2}{*}{\multicolumn{2}{|c|}{$\begin{array}{l}39.7 \\
48.5\end{array}$}} & \multicolumn{2}{|c|}{$29 \cdot 8$} & \multicolumn{2}{|c|}{$33 \cdot 3$} \\
\hline Ex & & & \multirow{2}{*}{\multicolumn{2}{|c|}{$\begin{array}{l}58.8 \\
11.5\end{array}$}} & & \\
\hline Current & \multicolumn{2}{|c|}{11.8} & & & \multicolumn{2}{|c|}{$\begin{array}{l}56.9 \\
9.76\end{array}$} \\
\hline $\operatorname{BMI}\left(\mathrm{kg} / \mathrm{m}^{2}\right)$ & 25.5 & 3.57 & $27 \cdot 7$ & 4.23 & 28.6 & 4.59 \\
\hline Physical activity (h/week) & $15 \cdot 0$ & $8 \cdot 15$ & $14 \cdot 3$ & $7 \cdot 79$ & $13 \cdot 1$ & $7 \cdot 34$ \\
\hline \multicolumn{7}{|l|}{ Dietary intake } \\
\hline Energy (kJ/d) & 9347 & 2586 & 9439 & 2548 & 9096 & 2502 \\
\hline Energy (kcal/d) & 2234 & 618 & 2256 & 609 & 2174 & 598 \\
\hline Carbohydrates, total (g/d) & 243 & $75 \cdot 3$ & 238 & $70 \cdot 0$ & 232 & $68 \cdot 1$ \\
\hline Proteins, total $(\mathrm{g} / \mathrm{d})$ & $86 \cdot 2$ & $22 \cdot 9$ & 87.4 & 21.9 & 83.8 & $21 \cdot 1$ \\
\hline Fats, total $(\mathrm{g} / \mathrm{d})$ & $87 \cdot 7$ & 30.6 & $90 \cdot 1$ & 32.5 & 84.7 & $29 \cdot 3$ \\
\hline Vegetables (g/d) & 191 & 105 & 193 & $99 \cdot 0$ & 176 & $93 \cdot 3$ \\
\hline Fruits $(g / d)$ & 188 & 140 & 178 & 143 & 172 & 138 \\
\hline Meat $(g / d)$ & $99 \cdot 2$ & 54.1 & 117 & $60 \cdot 4$ & 112 & $50 \cdot 8$ \\
\hline Fish $(g / d)$ & 21.8 & 18.9 & $22 \cdot 1$ & $19 \cdot 3$ & $21 \cdot 1$ & 17.9 \\
\hline \multicolumn{7}{|l|}{ Biological } \\
\hline Systolic blood pressure $(\mathrm{mmHg})$ & 131 & $17 \cdot 2$ & 138 & $17 \cdot 1$ & 143 & $19 \cdot 0$ \\
\hline Diastolic blood pressure $(\mathrm{mmHg})$ & $75 \cdot 2$ & 9.89 & 77.9 & 9.58 & $79 \cdot 1$ & $10 \cdot 3$ \\
\hline Cholesterol $(\mathrm{mmol} / \mathrm{l})$ & $5 \cdot 57$ & 1.03 & $5 \cdot 46$ & 1.15 & $5 \cdot 32$ & $1 \cdot 24$ \\
\hline $\mathrm{HDL}(\mathrm{mmol} / \mathrm{l})$ & 1.66 & 0.49 & 1.50 & 0.42 & 1.36 & 0.38 \\
\hline LDL (mmol/l) & 3.37 & 0.94 & $3 \cdot 26$ & 1.03 & $3 \cdot 16$ & $1 \cdot 14$ \\
\hline TAG (mmol/l) & 1.22 & 0.66 & 1.61 & 1.08 & 1.91 & 1.09 \\
\hline Medical history of CVD (\% yes) & \multicolumn{2}{|c|}{$12 \cdot 3$} & \multicolumn{2}{|c|}{14.1} & \multicolumn{2}{|c|}{23.4} \\
\hline
\end{tabular}

NGM, normal glucose metabolism; IGM, impaired glucose metabolism; ND T2DM, newly diagnosed type 2 diabetes mellitus.

in the highest intake tertile of fermented, skimmed and semiskimmed products and total dairy products (data not shown).

Associations between consumption of dairy products with the presence of impaired glucose metabolism or newly diagnosed type 2 diabetes mellitus

Age- and sex-adjusted analyses revealed significant inverse associations of total dairy, skimmed and fermented dairy products, as well as of cheese (both for total cheese and Dutch cheese) and yogurt, with IGM. After further adjustment for BMI, physical activity, smoking status, education and intakes of energy, vegetables, fruits, meat and fish, the associations of total dairy product intake and cheese with IGM were no longer statistically significant. The other associations remained significant, with OR, comparing the third with the first tertile, of 0.73 (95\% CI $0.55,0.96$ ) for skimmed products, 0.74 (95\% CI $0.54,0.99)$ for fermented products and 0.67 (95\% CI 0.50, 0.90) for yogurt. In continuous analyses, the OR per $100 \mathrm{~g}$ increment in fermented products and per serving of yogurt were only statistically significant in the age- and sex-adjusted models, whereas fully adjusted OR was 0.88 (95\% CI $0.80,0.97)$ per serving ( $20 \mathrm{~g}$ increment) of Dutch cheese consumption (Table 4). Semi-skimmed, full-fat and non-fermented products, as well as milk and curd cheese, were not associated with IGM in any of the models (Table 3).

Age- and sex-adjusted analyses of the association between dairy products and ND T2DM revealed significant inverse associations for total dairy products, semi-skimmed products, fermented products and yogurt, whereas full-fat products were positively associated with ND T2DM. Fully adjusted models comparing the third with the first tertile revealed OR of 0.50 (95\% CI $0.26,0.93)$ for total dairy product intake, but an increased OR of $2 \cdot 01$ (95\% CI 1.16, 3.47) for full-fat dairy products. Even though fully adjusted OR comparing the third $v$. the first tertile were not significant for total dairy product intake, fermented products and yogurt, the fully adjusted continuous models showed OR of 0.76 (95\% CI 0.61, 0.95) per $100 \mathrm{~g}$ increment in total dairy product intake, 0.69 (95\% CI 0.50, 0.94) per $100 \mathrm{~g}$ increment in fermented products and 0.47 (95\% CI $0 \cdot 24,0 \cdot 89)$ per serving $(150 \mathrm{ml})$ for yogurt (Table 4$)$, respectively. Non-fermented products, cheese and curd cheese were not associated with ND T2DM (Table 4).

Mutual adjustment for the associations of full-fat products with skimmed products and vice versa and of fermented products with non-fermented products did not alter the observed associations with IGM and ND T2DM.

\section{Discussion}

This is the first epidemiological study on the association of specific dairy products with both ND T2DM and IGM as outcomes. Detailed information on dairy product intake was collected using an extensive, 253-item, tailor-made FFQ that included fifty dairy items, and associations were extensively 
Table 3. Cross-sectional associations between intake of dairy products and impaired glucose metabolism (Odds ratios and $95 \%$ confidence intervals)*

\begin{tabular}{|c|c|c|c|c|c|c|c|c|}
\hline & \multicolumn{2}{|c|}{ Continuous } & \multirow{2}{*}{$\frac{\text { Tertile } 1}{\text { OR }}$} & \multicolumn{2}{|c|}{ Tertile 2} & \multicolumn{2}{|c|}{ Tertile 3} & \multirow[b]{2}{*}{$P_{\text {trend }}$} \\
\hline & OR & $95 \% \mathrm{Cl}$ & & OR & $95 \% \mathrm{Cl}$ & OR & $95 \% \mathrm{Cl}$ & \\
\hline Total dairy products (per $100 \mathrm{~g}$ ) & & & $\leq 87.5 \mathrm{~g} / \mathrm{d}$ & \multicolumn{2}{|c|}{$87.5-187.6 \mathrm{~g} / \mathrm{d}$} & \multicolumn{2}{|c|}{$\geq 187.6 \mathrm{~g} / \mathrm{d}$} & \\
\hline Model $1 \dagger$ & 0.91 & $0.84,0.99$ & 1 & 0.93 & $0.73,1.18$ & 0.76 & $0.59,0.97$ & 0.03 \\
\hline Model $2 \ddagger$ & 0.97 & $0.89,1.07$ & 1 & 1.07 & $0.81,1.42$ & 0.98 & $0.73,1.33$ & 0.92 \\
\hline Full-fat products (per $100 \mathrm{~g}$ ) & & & $\leq 4.26 \mathrm{~g} / \mathrm{d}$ & \multicolumn{2}{|c|}{$4.26-23.01 \mathrm{~g} / \mathrm{d}$} & \multicolumn{2}{|c|}{$\geq 23.01 \mathrm{~g} / \mathrm{d}$} & \\
\hline Model $1 \dagger$ & 0.91 & $0.68,1.22$ & 1 & 0.89 & $0.70,1 \cdot 14$ & 0.81 & $0.64,1.04$ & 0.09 \\
\hline Model $2 \ddagger$ & 0.99 & $0.70,1.40$ & 1 & 0.91 & $0.69,1.20$ & 0.90 & $0.67,1.21$ & 0.47 \\
\hline Semi-skimmed products (per $100 \mathrm{~g}$ ) & & & $\leq 2.75 \mathrm{~g} / \mathrm{d}$ & \multicolumn{2}{|c|}{$2.75-64.13 \mathrm{~g} / \mathrm{d}$} & \multicolumn{2}{|c|}{$\geq 64.13 \mathrm{~g} / \mathrm{d}$} & \\
\hline Model $1 \dagger$ & 0.92 & $0.81,1.03$ & 1 & 0.93 & $0.73,1.18$ & 0.80 & $0.62,1.02$ & 0.07 \\
\hline Model $2 \ddagger$ & 1.00 & $0.88,1.13$ & 1 & 0.92 & $0.69,1.23$ & 0.96 & $0.72,1.27$ & 0.76 \\
\hline Skimmed products (per $100 \mathrm{~g}$ ) & & & $\leq 6.50 \mathrm{~g} / \mathrm{d}$ & \multicolumn{2}{|c|}{$6.50-43.48 \mathrm{~g} / \mathrm{d}$} & \multicolumn{2}{|c|}{$\geq 43.48 \mathrm{~g} / \mathrm{d}$} & \\
\hline Model 1† & 0.93 & $0.81,1.06$ & 1 & 0.81 & $0.64,1.04$ & 0.77 & $0.60,0.98$ & 0.03 \\
\hline Model $2 \ddagger$ & 0.94 & $0.82,1.07$ & 1 & 0.72 & $0.55,0.96$ & 0.73 & $0.55, .96$ & 0.04 \\
\hline Fermented products (per $100 \mathrm{~g}$ ) & & & $\leq 61.2 \mathrm{~g} / \mathrm{d}$ & \multicolumn{2}{|c|}{$61.2-135.6 \mathrm{~g} / \mathrm{d}$} & \multicolumn{2}{|c|}{$\geq 135.6 \mathrm{~g} / \mathrm{d}$} & \\
\hline Model $1 \dagger$ & 0.85 & $0.76,0.95$ & 1 & 0.65 & $0.51,0.83$ & 0.62 & $0.48,0.79$ & $<0.01$ \\
\hline Model $2 \ddagger$ & 0.92 & $0.81,1.04$ & 1 & 0.69 & $0.52,0.92$ & 0.74 & $0.54,0.99$ & 0.04 \\
\hline Non-fermented products, basic (per $100 \mathrm{~g}$ ) & & & $\leq 1.13 \mathrm{~g} / \mathrm{d}$ & \multicolumn{2}{|c|}{$1.13-35.1 \mathrm{~g} / \mathrm{d}$} & \multicolumn{2}{|c|}{$\geq 35.1 \mathrm{~g} / \mathrm{d}$} & \\
\hline Model 1† & 0.96 & $0.85,1.09$ & 1 & 0.97 & $0.76,1.24$ & 1.00 & $0.78,1.24$ & 0.99 \\
\hline Model $2 \ddagger$ & 1.00 & $0.88,1.14$ & 1 & 0.90 & $0.68,1.21$ & 1.05 & $0.78,1.21$ & 0.72 \\
\hline Non-fermented products, desserts (per $100 \mathrm{~g}$ ) & & & $\leq 5.84 \mathrm{~g} / \mathrm{d}$ & \multicolumn{2}{|c|}{$5.84-26.07 \mathrm{~g} / \mathrm{d}$} & \multicolumn{2}{|c|}{$\geq 26.07 \mathrm{~g} / \mathrm{d}$} & \\
\hline Model $1 \dagger$ & 1.11 & $0.88,1.39$ & 1 & $1 \cdot 10$ & $0.86,1.40$ & 0.90 & $0.70,1.15$ & 0.43 \\
\hline Model $2 \ddagger$ & 1.22 & $0.94,1.59$ & 1 & 1.14 & $0.86,1.52$ & 0.99 & $0.73,1.35$ & 0.96 \\
\hline Milk (per serving $(150 \mathrm{~g})$ ) & & & Non-cons & \multicolumn{2}{|c|}{$0.1-43.5 \mathrm{~g} / \mathrm{d}$} & \multicolumn{2}{|c|}{$\geq 43.5 \mathrm{~g} / \mathrm{d}$} & \\
\hline Model $1 \dagger$ & 0.94 & $0.73,1.21$ & 1 & 1.01 & $0.77,1.31$ & 1.10 & $0.86,1.41$ & 0.08 \\
\hline Model $2 \ddagger$ & 1.01 & $0.77,1.31$ & 1 & 0.89 & $0.66,1.20$ & $1 \cdot 10$ & $0.84,1.45$ & 0.54 \\
\hline Cheese, total (per serving $(20 \mathrm{~g})$ ) & & & $\leq 17.5 \mathrm{~g} / \mathrm{d}$ & \multicolumn{2}{|c|}{$17.5-39.8 \mathrm{~g} / \mathrm{d}$} & \multicolumn{2}{|c|}{$\geq 39.8 \mathrm{~g} / \mathrm{d}$} & \\
\hline Model 1† & 0.89 & $0.82,0.97$ & 1 & 0.95 & $0.75,1.21$ & 0.67 & $0.52,0.87$ & $<0.01$ \\
\hline Model $2 \ddagger$ & 0.89 & $0.81,0.97$ & 1 & 1.04 & $0.79,1.36$ & 0.75 & $0.55,1.01$ & 0.07 \\
\hline Cheese, Dutch (per serving $(20 \mathrm{~g})$ ) & & & $\leq 15.4 \mathrm{~g} / \mathrm{d}$ & \multicolumn{2}{|c|}{$15.4-34.3 \mathrm{~g} / \mathrm{d}$} & \multicolumn{2}{|c|}{$\geq 34.4 \mathrm{~g} / \mathrm{d}$} & \\
\hline Model $1 \dagger$ & 0.89 & $0.81,0.97$ & 1 & 0.88 & $0.69,1.12$ & 0.69 & $0.54,0.88$ & $<0.01$ \\
\hline Model $2 \ddagger$ & 0.88 & $0.80,0.97$ & 1 & 0.93 & $0.70,1.23$ & 0.76 & $0.57,1.01$ & 0.06 \\
\hline Yogurt, total (per serving $(150 \mathrm{~g})$ ) & & & $\leq 10.5 \mathrm{~g} / \mathrm{d}$ & \multicolumn{2}{|c|}{$13.5-63 \mathrm{~g} / \mathrm{d}$} & & $\mathrm{g} / \mathrm{d}$ & \\
\hline Model $1 \dagger$ & 0.77 & $0.61,0.96$ & 1 & 0.73 & $0.57,0.94$ & 0.61 & $0.48,0.78$ & $<0.01$ \\
\hline Model $2 \ddagger$ & 0.90 & $0.71,1.15$ & 1 & 0.72 & $0.54,0.97$ & 0.67 & $0.50,0.90$ & $<0.01$ \\
\hline Curd cheese, total (per serving $(150 \mathrm{~g})$ ) & & & Non-cons & & $g / d$ & & $g / d$ & \\
\hline Model 1† & 0.96 & $0.62,1.46$ & 1 & 0.85 & $0.65,1.10$ & 0.90 & $0.70,1.16$ & 0.71 \\
\hline Model $2 \ddagger$ & 1.05 & $0.67,1.64$ & 1 & 1.01 & $0.76,1.36$ & 0.94 & $0.71,1.25$ & 0.42 \\
\hline
\end{tabular}

Non-cons, non-consumers.

* Continuous analyses: milk, cheese, yogurt and curd cheese per serving increment, all combined dairy categories per $100 \mathrm{~g}$ increment

† Model 1 adjusted for age and sex.

‡ Model 2 additionally adjusted for BMI, physical activity, smoking status, education and intakes of energy, vegetables, fruits, meat and fish.

adjusted for potential confounders. To prevent reporting bias, participants filled out the FFQ before results of the OGTT became available, and only individuals with ND T2DM (screen-diagnosed diabetes) were included. Results showed a strong inverse association between total dairy product intake and ND T2DM, with an OR of 0.50 (95\% CI $0 \cdot 26,0 \cdot 93$ ) for the highest $v$. lowest tertile of intake, but a marked positive association between full-fat dairy products and T2DM (OR 2.01; 95\% CI 1.16, 3.47). Remarkably, these associations were completely absent for IGM (OR 0.98 for the highest tertile $v$. lowest of total dairy and 0.90 for full-fat products). Fermented products and yogurt were inversely associated with T2DM $(\mathrm{OR}<0.60)$ and the same was observed for IGM $(\mathrm{OR}<0.75)$. An inverse association with IGM was found for skimmed dairy products (OR for the top $v$. bottom tertile of intake: 0.73 (95\% CI $0.55,0.96)$ ), which was non-significant for ND T2DM despite a similar OR (0.76 (95\% CI 0.44, 1.32)). Finally, the consumption of cheese showed a significant negative association with IGM (OR per serving (20 g): 0.88 (95\% CI 0.80, 0.97)) but not with T2DM (OR per serving: 1.05 (95\% CI 0.90, 1.22)).
Four meta-analyses ${ }^{(8,10-12)}$ revealed combined hazard ratio of $0 \cdot 85^{(8)}$ and $0 \cdot 86^{(12)}$ for highest $v$. lowest categories of total dairy consumption $-0 \cdot 80^{(11)}$ to $0 \cdot 93^{(10)}$ for 200 and $400 \mathrm{~g}$ increment of total dairy product intake ${ }^{(11)}$, respectively. Our results confirmed this inverse association, but associations in our study were more pronounced with an estimated $50 \%$ lower odds of T2DM in individuals in the third tertile (total dairy product intake $>188 \mathrm{~g} / \mathrm{d}$ ) as compared with individuals in the first tertile (total dairy product intake $<87 \mathrm{~g} / \mathrm{d}$ ). Published studies also showed inverse associations of both skimmed and full-fat dairy products ${ }^{(14)}$ with T2DM. The present study showed a positive association of full-fat products with ND T2DM, but not with IGM. In addition, skimmed products were significantly inversely associated with IGM, but not with ND T2DM. This is in contrast with a recent prospective study showing an inverse association of full-fat dairy products with T2DM risk and no association between skimmed products and T2DM risk ${ }^{(20)}$. Several other studies showed a negative association of skimmed products with T2DM risk but no association of full-fat dairy products with 
Table 4. Cross-sectional associations between intake of dairy products and newly diagnosed type 2 diabetes mellitus (Odds ratios and $95 \%$ confidence intervals)*

\begin{tabular}{|c|c|c|c|c|c|c|c|c|}
\hline & \multicolumn{2}{|c|}{ Continuous } & \multirow{2}{*}{$\frac{\text { Tertile } 1}{\text { OR }}$} & \multicolumn{2}{|c|}{ Tertile 2} & \multicolumn{2}{|c|}{ Tertile 3} & \multirow[b]{2}{*}{$P_{\text {trend }}$} \\
\hline & OR & $95 \% \mathrm{Cl}$ & & OR & $95 \% \mathrm{Cl}$ & OR & $95 \% \mathrm{Cl}$ & \\
\hline Total dairy & & & $\leq 87.5 \mathrm{~g} / \mathrm{d}$ & \multicolumn{2}{|c|}{$87.5-187.6 \mathrm{~g} / \mathrm{d}$} & \multicolumn{2}{|c|}{$\geq 187.6 \mathrm{~g} / \mathrm{d}$} & \\
\hline Model $1 \dagger$ & 0.74 & $0.61,0.88$ & 1 & 1.17 & $0.78,1 \cdot 74$ & 0.42 & $0.25,0.71$ & $<0.01$ \\
\hline Model $2 \ddagger$ & 0.76 & $0.61,0.95$ & 1 & 1.38 & $0.86,2.24$ & 0.50 & $0.26,0.93$ & 0.06 \\
\hline Full-fat products & & & $\leq 4.26 \mathrm{~g} / \mathrm{d}$ & \multicolumn{2}{|c|}{$4.26-23.01 \mathrm{~g} / \mathrm{d}$} & \multicolumn{2}{|c|}{$\geq 23.01 \mathrm{~g} / \mathrm{d}$} & \\
\hline Model 1† & $1 \cdot 14$ & $0.71,1.83$ & 1 & $1 \cdot 16$ & $0.73,1.85$ & 1.50 & $0.87,2.33$ & 0.07 \\
\hline Model 2‡ & 1.60 & $0.93,2.76$ & 1 & 1.32 & $0.77,2 \cdot 28$ & $2 \cdot 01$ & $1.16,3.47$ & 0.01 \\
\hline Semi-skimmed products & & & $\leq 2.75 \mathrm{~g} / \mathrm{d}$ & \multicolumn{2}{|c|}{$2.75-64.13 \mathrm{~g} / \mathrm{d}$} & \multicolumn{2}{|c|}{$\geq 64.13 \mathrm{~g} / \mathrm{d}$} & \\
\hline Model $1 \dagger$ & 0.71 & $0.54,0.94$ & 1 & 0.96 & $0.63,1.46$ & 0.58 & $0.37,0.93$ & 0.03 \\
\hline Model $2 \ddagger$ & 0.75 & $0.55,1.02$ & 1 & 0.85 & $0.52,1.40$ & 0.60 & $0.35,1.02$ & 0.06 \\
\hline \multicolumn{9}{|l|}{ Skimmed products } \\
\hline Model 1† & 0.72 & $0.52,0.99$ & 1 & 0.95 & $0.62,1.45$ & 0.83 & $0.52,1.30$ & 0.42 \\
\hline Model $2 \ddagger$ & 0.71 & $0.48,1.04$ & 1 & 0.85 & $0.51,1.41$ & 0.76 & $0.44,1.32$ & 0.32 \\
\hline Fermented products & & & $\leq 61.2 \mathrm{~g} / \mathrm{d}$ & & $5.6 \mathrm{~g} / \mathrm{d}$ & & $\mathrm{g} / \mathrm{d}$ & \\
\hline Model 1† & 0.59 & $0.45,0.77$ & 1 & 0.64 & $0.42,0.98$ & 0.42 & $0.26,0.68$ & $<0.01$ \\
\hline Model $2 \ddagger$ & 0.69 & $0.50,0.94$ & 1 & 0.76 & $0.46,1.26$ & 0.57 & $0.32,1.01$ & 0.05 \\
\hline Non-fermented products, basic & & & $\leq 1.13 \mathrm{~g} / \mathrm{d}$ & \multicolumn{2}{|c|}{$1.13-35.1 \mathrm{~g} / \mathrm{d}$} & \multicolumn{2}{|c|}{$\geq 35.1 \mathrm{~g} / \mathrm{d}$} & \\
\hline Model $1 \dagger$ & 0.84 & $0.66,1.07$ & 1 & 1.09 & $0.70,1.69$ & 1.05 & $0.67,1.65$ & 0.82 \\
\hline Model $2 \ddagger$ & 0.80 & $0.60,1.07$ & 1 & 0.89 & $0.53,1.50$ & 0.85 & $0.50,1.45$ & 0.56 \\
\hline Non-fermented products, desserts & & & $\leq 5.84 \mathrm{~g} / \mathrm{d}$ & \multicolumn{2}{|c|}{$5.84-26.07 \mathrm{~g} / \mathrm{d}$} & \multicolumn{2}{|c|}{$\geq 26.07 \mathrm{~g} / \mathrm{d}$} & \\
\hline Model 1† & 0.75 & $0.46,1.21$ & 1 & 1.44 & $0.92,2.24$ & 1.03 & $0.65,1.64$ & 0.89 \\
\hline Model $2 \ddagger$ & 0.78 & $0.44,1.38$ & 1 & 1.59 & $0.94,2.71$ & 1.14 & $0.64,2.02$ & 0.71 \\
\hline Milk (per serving $(150 \mathrm{~g})$ ) & & & Non-cons & \multicolumn{2}{|c|}{$0.1-43.5 \mathrm{~g} / \mathrm{d}$} & \multicolumn{2}{|c|}{$\geq 43.5 \mathrm{~g} / \mathrm{d}$} & \\
\hline Model $1 \dagger$ & 0.68 & $0.41,1.15$ & 1 & 1.20 & $0.77,1.87$ & 0.92 & $0.58,1.45$ & 0.74 \\
\hline Model $2 \ddagger$ & 0.62 & $0.33,1.15$ & 1 & $1 \cdot 16$ & $0.70,1.92$ & 0.81 & $0.48,1.39$ & 0.53 \\
\hline Cheese, total (per serving $(20 \mathrm{~g})$ ) & & & $\leq 17.5 \mathrm{~g} / \mathrm{d}$ & \multicolumn{2}{|c|}{$17.5-39.8 \mathrm{~g} / \mathrm{d}$} & \multicolumn{2}{|c|}{$\geq 39.8 \mathrm{~g} / \mathrm{d}$} & \\
\hline Model 1† & 0.96 & $0.84,1.09$ & 1 & 0.88 & $0.57,1.37$ & 0.89 & $0.57,1.39$ & 0.61 \\
\hline Model $2 \ddagger$ & 1.03 & $0.89,1.18$ & 1 & 0.94 & $0.56,1.58$ & 1.15 & $0.68,1.97$ & 0.60 \\
\hline Cheese, Dutch (per serving $(20 \mathrm{~g})$ ) & & & $\leq 15.4 \mathrm{~g} / \mathrm{d}$ & \multicolumn{2}{|c|}{$15.4-34.3 \mathrm{~g} / \mathrm{d}$} & \multicolumn{2}{|c|}{$\geq 34.4 \mathrm{~g} / \mathrm{d}$} & \\
\hline Model $1 \dagger$ & 0.99 & $0.86,1.13$ & 1 & 0.97 & $0.61,1.53$ & 0.98 & $0.63,1.51$ & 0.91 \\
\hline Model $2 \ddagger$ & 1.05 & $0.90,1.22$ & 1 & 1.07 & $0.62,1.83$ & 1.26 & $0.74,2.13$ & 0.39 \\
\hline Yogurt, total (per serving $(150 \mathrm{~g})$ ) & & & $\leq 10.5 \mathrm{~g} / \mathrm{d}$ & \multicolumn{2}{|c|}{$13.5-63 \mathrm{~g} / \mathrm{d}$} & \multicolumn{2}{|c|}{$\geq 63 \mathrm{~g} / \mathrm{d}$} & \\
\hline Model $1 \dagger$ & 0.36 & $0.21,0.64$ & 1 & 0.79 & $0.51,1.23$ & 0.54 & $0.35,0.85$ & $<0.01$ \\
\hline Model $2 \ddagger$ & 0.47 & $0.24,0.89$ & 1 & 0.87 & $0.52,1.45$ & 0.60 & $0.35,1.02$ & 0.06 \\
\hline Curd cheese, total (per serving $(150 \mathrm{~g})$ ) & & & Non-cons & \multicolumn{2}{|c|}{$0.1-9 \mathrm{~g} / \mathrm{d}$} & \multicolumn{2}{|c|}{$\geq 9 \mathrm{~g} / \mathrm{d}$} & \\
\hline Model $1 \dagger$ & 0.34 & $0.09,1.30$ & 1 & 0.57 & $0.34,0.95$ & 0.67 & $0.42,1.06$ & 0.05 \\
\hline Model $2 \ddagger$ & 0.49 & $0.33,0.72$ & 1 & 0.74 & $0.42,1.31$ & 0.79 & $0.46,1.36$ & 0.15 \\
\hline
\end{tabular}

Non-cons, non-consumers.

* Continuous analyses: milk, cheese, yogurt and curd cheese per serving increment, all combined dairy categories per $100 \mathrm{~g}$ increment

$\dagger$ Model 1 adjusted for age and sex.

¥ Model 2 additionally adjusted for BMI, physical activity, smoking status, education and intakes of energy, vegetables, fruits, meat and fish.

T2DM risk ${ }^{(32,33)}$. Although it is unclear why full-fat products were positively associated with T2DM in our study, but not with IGM, one possible reason may have been the relatively low number of individuals with ND T2DM ( $n$ 125), which may have led to a false-positive finding. In addition, the heterogeneity of the IGM population may have attenuated the association, which may explain this difference. Future studies need to further investigate associations of skimmed and full-fat dairy products with IGM and T2DM risk.

There is very less information on the association between single dairy products and T2DM risk. Meta-analyses and a recent study have shown that milk consumption was inversely ${ }^{(8)}$ or not associated with the risk of T2DM in fully adjusted models ${ }^{(10-12,20)}$. In our study, the null finding for milk consumption was confirmed for both T2DM and IGM. Although some studies did not reveal an association of cheese consumption with the incidence of the metabolic syndrome and/or $\mathrm{T}_{2} \mathrm{DM}^{(13,20)}$, a meta-analysis showed an $8^{(10)}$ to $20 \%^{(11)}$ reduction in relative risk per $50^{(10)}$ or $30 \mathrm{~g}^{(11)}$ increment in cheese consumption. In our population, cheese consumption was not associated with ND T2DM, but a significantly lower odds of IGM of $11 \%$ was observed per serving $(20 \mathrm{~g})$ of cheese, which corresponded with a lower odds of IGM of 26 and $16 \%$ per 50 and $30 \mathrm{~g}$ increment (data not shown), respectively. In line with a potential inverse association of cheese consumption on diabetes risk, a recent randomised controlled trial showed significant decreases in total cholesterol and waist circumference after increased cheese consumption for a period of 8 weeks in individuals with the metabolic syndrome ${ }^{(34)}$. Our finding that consumption of yogurt and fermented products was inversely associated with both T2DM and IGM is also consistent with previous studies $^{(10-12,18)}$. In line with this, a cross-sectional study performed within the Framingham Heart Study Offspring and Third Generation cohorts revealed that individuals who consumed yogurt had a better diet quality as compared with nonconsumers. In addition, yogurt consumption was associated with lower circulating levels of glucose and TAG, as well as with lower systolic blood pressure and insulin resistance ${ }^{(35)}$. 
Furthermore, fermentation may enhance the nutritional value of yogurt and provide it with unique properties that enhance the bioavailability of some nutrients ${ }^{(36)}$ and reduce blood pressure and LDL-cholesterol ${ }^{(37)}$. In contrast, the recently published Seguimiento Universidad de Navarra (SUN) study revealed no associations between yogurt consumption and the incidence of the metabolic syndrome during a 6-year follow-up period ${ }^{(38)}$.

The mechanisms underlying alleged protective effects of total dairy products or fermented products on T2DM are not well understood. It has been hypothesised that dairy product intake may be inversely associated with T2DM through a protective role of vitamin $\mathrm{D}, \mathrm{Ca}, \mathrm{Mg}$ and whey proteins present in dairy products $^{(39)}$. As dairy products in the Netherlands are not fortified with vitamin $\mathrm{D}$, this vitamin is unlikely to play a role in the present study results. Ca increases insulin secretion and is essential for insulin-responsive tissues such as skeletal muscle and adipose tissue and may reduce insulin resistance ${ }^{(40)}$. Furthermore, Mg intake has been associated with reduced diabetes risk in epidemiological studies ${ }^{(41)}$ and with improved insulin sensitivity in some experimental studies, although data are limited ${ }^{(42)}$. Dairy products also contain whey proteins, which in animal models have been shown to reduce body weight gain and to increase insulin sensitivity ${ }^{(43)}$. In contrast with these potentially protective nutrients, dairy products are also rich sources of SFA, which were associated with insulin resistance in some but not all controlled trials and prospective studies ${ }^{(44)}$. In the recent EPIC-InterAct study, plasma levels of even-chain SFA were positively associated with incident T2DM, whereas odd-chain SFA were inversely associated with incident $\mathrm{T}_{2} \mathrm{DM}^{(45)}$. As SFA can be synthesised in the human body, the physiological implication of this finding remains to be elucidated. Two studies revealed that higher plasma levels of trans-palmitoleic acid, which is indicative of dairy fat consumption, were associated with a reduced diabetes risk ${ }^{(5,15)}$. In addition, animal studies showed that butyrate consumption, one of the main fatty acids in dairy products, could protect from obesity and increase insulin sensitivity $^{(7)}$.

It is possible that the observed inverse association of Dutch cheese, yogurt, skimmed and fermented products with IGM and of total dairy product intake and high-fat dairy product intake with ND T2DM reflects a general difference in lifestyle habits. For instance, in our study population, higher intake of total dairy products was associated with healthier lifestyle reflected by higher levels of physical activity, lower prevalence of smoking and overweight/obesity, lower intakes of meat and higher intakes of fruits and vegetables. Even though associations between specific dairy products remained significant after adjustment for these variables, the possibility of residual confounding cannot be fully disregarded.

Our study has several strengths and limitations. A major limitation is the cross-sectional design of our study, which formally rules out causal inference. Nevertheless, we believe that our analyses do add to the existing evidence on the relationship between dairy product intake and $\mathrm{T} 2 \mathrm{DM}$, as all participants completed the FFQ before being informed about their glucose metabolism status, which minimised the risk of reporting bias. Furthermore, subjects with known pre-existing T2DM ( $n$ 883) were excluded from the present analyses, which makes it quite unlikely that subjects with ND T2DM could have adapted their diet to their disease status.

It may be noted that our results are generally in line with associations observed in previous prospective studies, which corroborates the integrity of our findings. A strength of our study is the fact that we have reported unique data on IGM, which have not been published before. Nevertheless, of note, IGM is a mixed population defined as the presence of either an elevated fasting glucose level, an IGT test or the combination of both. Furthermore, extensive information on lifestyle factors was collected for each cohort member, allowing the adjustment for potential confounders. Another important strength of this study is the extensive collection of dairy product intake data. We believe that the FFQ used in the Maastricht Study optimally estimated dairy product intake as our extensive FFQ captured fifty dairy food items.

In conclusion, this is the first study on the associations of dairy products with both ND T2DM and IGM. Intakes in the top tertiles of yogurt and fermented products were cross-sectionally associated with lower odds of presenting ND T2DM and IGM by $25-40 \%$ relatively to intakes in the bottom tertiles. This observed association is consistent with the hypothesis that yogurt and fermented products may play a role in the aetiology of diabetes. However, associations of total dairy product intake, full-fat products, skimmed products and Dutch cheese did not reveal similar direction and magnitude for ND T2DM and IGM. Therefore, our results warrant further assessment of the relationship between single dairy products and the incidence of IGM and T2DM in a prospective design to elucidate their role in T2DM development.

\section{Acknowledgements}

The authors thank all the voluntary participants from the Maastricht Study as well as the funding bodies.

The Maastricht Study is supported by the European Regional Development Fund as part of OP-ZUID, the province of Limburg, the Department of Economic Affairs of the Netherlands (grant no. 31O.041), Stichting the Weijerhorst, the Pearl String Initiative Diabetes, the Cardiovascular Center Maastricht, Cardiovascular Research Institute Maastricht (CARIM), School for Nutrition, Toxicology and Metabolism (NUTRIM), Stichting Annadal, Health Foundation Limburg and by unrestricted grants from Janssen, Novo Nordisk and Sanofi. Furthermore, the authors thank FrieslandCampina for their financial support of the current study.

The contributions of the authors are as follows: M. T. S., S. J. S. S., C. J. v. d. K., A. K., N. S., R. M. A. H., C. D. A. S. and P. C. D. participated in the design and implementation of the Maastricht Study. M. C. J. M. v. D., N. W., L. d. B., S. J. P. M. E. and P. C. D. collected and cleaned the dietary intake data. S. J. P. M. E. and P. C. D. performed data analyses and wrote the initial draft of the manuscript. S. J. P. M. E., M. C. J. M. v. D., N. W., L. d. B., S. J. W. H. O. E., C. M. S.-P., M. T. S., S. J. S. S., C. J. v. d. K., A. K., N. S., R. M. A. H., C. D. A. S. and P. C. D. critically read the manuscript and approved the final version. 
S. J. W. H. O. E. and C. M. S.-P. are employees at FrieslandCampina. All the other authors have no potential conflicts of interest to declare.

\section{Supplementary material}

For supplementary material/s referred to in this article, please visit http://dx.doi.org/doi:10.1017/S0007114516000313

\section{References}

1. Guariguata L, Whiting D, Weil C, et al. (2011) The International Diabetes Federation diabetes atlas methodology for estimating global and national prevalence of diabetes in adults. Diabetes Res Clin Pract 94, 322-332.

2. Zimmet P (2003) The burden of type 2 diabetes: are we doing enough? Diabetes Metab 29, 6S9-18.

3. Carpentier A, Mittelman SD, Bergman RN, et al. (2000) Prolonged elevation of plasma free fatty acids impairs pancreatic beta-cell function in obese nondiabetic humans but not in individuals with type 2 diabetes. Diabetes $\mathbf{4 9}$, 399-408.

4. Kashyap SR, Belfort R, Berria R, et al. (2004) Discordant effects of a chronic physiological increase in plasma FFA on insulin signaling in healthy subjects with or without a family history of type 2 diabetes. Am J Physiol Endocrinol Metab 287, E537-E546.

5. Mozaffarian D, de Oliveira Otto MC, Lemaitre RN, et al. (2013) trans-Palmitoleic acid, other dairy fat biomarkers, and incident diabetes: the Multi-Ethnic Study of Atherosclerosis (MESA). Am J Clin Nutr 97, 854-861.

6. Krachler B, Norberg M, Eriksson JW, et al. (2008) Fatty acid profile of the erythrocyte membrane preceding development of type 2 diabetes mellitus. Nutr Metab Cardiovascu Dis 18, 503-510.

7. den Besten G, Bleeker A, Gerding A, et al. (2015) Short-chain fatty acids protect against high-fat diet-induced obesity via a PPAR gamma-dependent switch from lipogenesis to fat oxidation. Diabetes 64, 2389-2408.

8. Elwood PC, Givens DI, Beswick AD, et al. (2008) The survival advantage of milk and dairy consumption: an overview of evidence from cohort studies of vascular diseases, diabetes and cancer. J Am Coll Nutr 27, 723S-734S.

9. Kalergis M, Leung Yinko SS \& Nedelcu R (2013) Dairy products and prevention of type 2 diabetes: implications for research and practice. Front Endocrinol (Lausanne) 4, 90.

10. Aune D, Norat T, Romundstad P, et al. (2013) Dairy products and the risk of type 2 diabetes: a systematic review and doseresponse meta-analysis of cohort studies. Am J Clin Nutr $\mathbf{9 8}$, 1066-1083.

11. Gao D, Ning N, Wang C, et al. (2013) Dairy products consumption and risk of type 2 diabetes: systematic review and dose-response meta-analysis. PLOS ONE 8, e73965.

12. Tong X, Dong JY, Wu ZW, et al. (2011) Dairy consumption and risk of type 2 diabetes mellitus: a meta-analysis of cohort studies. Eur J Clin Nutr 65, 1027-1031.

13. Fumeron F, Lamri A, Abi Khalil C, et al. (2011) Dairy consumption and the incidence of hyperglycemia and the metabolic syndrome: results from a french prospective study, Data from the Epidemiological Study on the Insulin Resistance Syndrome (DESIR). Diabetes Care 34, 813-817.

14. Malik VS, Sun Q, van Dam RM, et al. (2011) Adolescent dairy product consumption and risk of type 2 diabetes in middleaged women. Am J Clin Nutr 94, 854-861.
15. Mozaffarian D, Cao H, King IB, et al. (2010) trans-Palmitoleic acid, metabolic risk factors, and new-onset diabetes in U.S. adults: a cohort study. Ann Intern Med 153, 790-799.

16. Nikooyeh B, Neyestani TR, Farvid M, et al. (2011) Daily consumption of vitamin D- or vitamin D+calcium-fortified yogurt drink improved glycemic control in patients with type 2 diabetes: a randomized clinical trial. Am J Clin Nutr $\mathbf{9 3}$, $764-771$.

17. Stancliffe RA, Thorpe T \& Zemel MB (2011) Dairy attentuates oxidative and inflammatory stress in metabolic syndrome. Am J Clin Nutr 94, 422-430.

18. Diaz-Lopez A, Bullo M, Martinez-Gonzalez MA, et al. (2015) Dairy product consumption and risk of type 2 diabetes in an elderly Spanish Mediterranean population at high cardiovascular risk. Eur J Nutr 55, 349-360.

19. Sluijs I, Forouhi NG, Beulens JW, et al. (2012) The amount and type of dairy product intake and incident type 2 diabetes: results from the EPIC-InterAct Study. Am J Clin Nutr 96, 382-390.

20. Ericson U, Hellstrand S, Brunkwall L, et al. (2015) Food sources of fat may clarify the inconsistent role of dietary fat intake for incidence of type 2 diabetes. Am J Clin Nutr 101, 1065-1080

21. Hjartaker A, Lagiou A, Slimani N, et al. (2002) Consumption of dairy products in the European Prospective Investigation into Cancer and Nutrition (EPIC) cohort: data from 35955 24-hour dietary recalls in 10 European countries. Public Health Nutr $\mathbf{5}$, 1259-1271.

22. Tabak AG, Herder C, Rathmann W, et al. (2012) Prediabetes: a high-risk state for diabetes development. Lancet 379, 2279-2290.

23. Wild S, Roglic G, Green A, et al. (2004) Global prevalence of diabetes: estimates for the year 2000 and projections for 2030. Diabetes Care 27, 1047-1053.

24. Bullard KM, Saydah SH, Imperatore G, et al. (2013) Secular changes in U.S. prediabetes prevalence defined by hemoglobin A1c and fasting plasma glucose: National Health and Nutrition Examination Surveys, 1999-2010. Diabetes Care 36, 2286-2293

25. Hu FB (2011) Globalization of diabetes: the role of diet, lifestyle, and genes. Diabetes Care 34, 1249-1257.

26. Schram MT, Sep SJ, van der Kallen CJ, et al. (2014) The Maastricht Study: an extensive phenotyping study on determinants of type 2 diabetes, its complications and its comorbidities. Eur J Epidemiol 29, 439-451.

27. Nederlands Huisartsen Genootschap (2013) NHG-Standaard Diabetes mellitus type 2 (derde herziening) (NHG Standard Diabetes mellitus type 2 (third revision)). https://www.nhg. org/standaarden/volledig/nhg-standaard-diabetes-mellitustype-2 (accessed January 2016).

28. Willett WC (1998) Issues in analysis and presentation of dietary data. In Nutritional Epidemiology, 2nd ed., pp. 321-346 [WC Willett, editor]. New York, NY: Oxford University Press.

29. World Health Organization (2006) Definition and diagnosis of diabetes mellitus and intermediate hyperglycemia. http://www. whoint/diabetes/publications/Definition\%20and\%20diagnosis\% 20of\%20diabetes_newpdf (accessed January 2016).

30. Molag ML, de Vries JH, Duif N, et al. (2010) Selecting informative food items for compiling food-frequency questionnaires: comparison of procedures. Br J Nutr 104, 446-456.

31. National Institute for Public Health and the Environment (2011) Dutch Food Composition Database. Stichting NEVO N-ovR, Bilthoven. http://www.rivm.nl/en/Topics/D/Dutch_ Food_Composition_Database (accessed January 2016).

32. Choi HK, Willett WC, Stampfer MJ, et al. (2005) Dairy consumption and risk of type 2 diabetes mellitus in men: a prospective study. Arch Inter Med 165, 997-1003. 
33. Liu S, Choi HK, Ford E, et al. (2006) A prospective study of dairy intake and the risk of type 2 diabetes in women. Diabetes Care 29, 1579-1584.

34. Nilsen R, Hostmark AT, Haug A, et al. (2015) Effect of a high intake of cheese on cholesterol and metabolic syndrome: results of a randomized trial. Food Nutr Res 59, 27651.

35. Wang H, Livingston KA, Fox CS, et al. (2013) Yogurt consumption is associated with better diet quality and metabolic profile in American men and women. Nutr Res 33, 18-26.

36. Astrup A (2014) Yogurt and dairy product consumption to prevent cardiometabolic diseases: epidemiologic and experimental studies. Am J Clin Nutr 99, 1235s-1242s.

37. Agerholm-Larsen L, Raben A, Haulrik N, et al. (2000) Effect of 8 week intake of probiotic milk products on risk factors for cardiovascular diseases. Eur J Clin Nutr 54, 288-297.

38. Sayon-Orea C, Bes-Rastrollo M, Marti A, et al. (2015) Association between yogurt consumption and the risk of metabolic syndrome over 6 years in the SUN study. BMC Public Health 15, 170.

39. Weaver CM (2014) How sound is the science behind the dietary recommendations for dairy? Am J Clin Nutr 99, 1217s-1222s.
40. Pittas AG, Lau J, Hu FB, et al. (2007) The role of vitamin D and calcium in type 2 diabetes. A systematic review and meta-analysis. J Clin Endocrinol Metab 92, 2017-2029.

41. Dong JY, Xun P, He K, et al. (2011) Magnesium intake and risk of type 2 diabetes: meta-analysis of prospective cohort studies. Diabetes Care 34, 2116-2122.

42. Volpe SL (2008) Magnesium, the metabolic syndrome, insulin resistance, and type 2 diabetes mellitus. Crit Rev Food Sci Nutr 48, 293-300

43. Belobrajdic DP, McIntosh GH \& Owens JA (2004) A highwhey-protein diet reduces body weight gain and alters insulin sensitivity relative to red meat in wistar rats. $J$ Nutr $\mathbf{1 3 4}$, $1454-1458$.

44. Micha R \& Mozaffarian D (2010) Saturated fat and cardiometabolic risk factors, coronary heart disease, stroke, and diabetes: a fresh look at the evidence. Lipids 45, 893-905.

45. Forouhi NG, Koulman A, Sharp SJ, et al. (2014) Differences in the prospective association between individual plasma phospholipid saturated fatty acids and incident type 2 diabetes: the EPIC-InterAct case-cohort study. Lancet Diabetes Endocrinol 2, 810-818. 